\title{
Edge clamping effect on the fatigue resistance of an orthotropic steel bridge deck
}

\author{
Philippe Van Bogaert*,a \\ ${ }^{a}$ Ghent University, Dept. Civil Engineering, Belgium \\ philippe.vanbogaert@ugent.bemailto:
}

\begin{abstract}
Orthotropic steel decks are an excellent solution for long span bridges, except if the spreading of wheel loads through the pavement is poor. In the latter case they are heavily sensitive to fatigue cracking. The most critical locations of fatigue cracks are the connection of longitudinal stiffeners to the deck plate and the cutouts and cope-holes in the webs of the transverse stiffeners. OSD with variable depth are seldom used, since the idea contradicts with one of the main assets to limit the bridge deck depth. However, in few cases variable depth may be meaningful as in the case of a railway bridge across river Dyle, recently built in Mechelen (Belgium). This $50 \mathrm{~m}$ span bridge consists of 2 lateral stubs of $1.55 \mathrm{~m}$ diameter connected by the OSD. The tubes are slightly bent to foster arch thrust action and rotated towards the bridge axis. In addition, the arch springs are encased in the concrete abutment. Due to this arrangement, the crossbeams are partially clamped in the lateral tubes of high torsion stiffness. The effect of the end clamping of crossbeams reduces the tension in the lower part of the crossbeam web, also near the outer cope holes, where the largest stress concentrations occur and fatigue resistance is the lowest. The reduction of the magnitude of these stress concentrations is significant and depends on the crossbeam span. However, the effect disappears if the crossbeam web is higher, thus confirming it is due to bending tension of the crossbeams. In addition, the influence length of crossbeams has been verified in the case of river Dyle bridge and complies well with Eurocode.
\end{abstract}

Keywords: Orthotropic plated bridge deck, stress concentrations, cope holes in webs, steel tube arch bridge

\section{INTRODUCTION}

Orthotropic steel plated bridge decks (OSD) are used in all major long-span bridges and even in medium-span bridges. This type of deck has excellent capacity to reduce the total depth of a bridge superstructure and thus contributes to slenderness and building lighter structures. However, in the past and on occasions even today, fatigue damage has occurred to some OSD's. This is due to the multiple details, both welding and geometric, appearing in orthotropic plates and is in contrast with the high robustness of these structures, obtained through membrane action of the deck plate.

Most OSD's consist of closed-section stiffeners, fabricated by cold-bending of flat plates and welded to the deck plate by the shielded arc process. This no longer requires any preparation of the stiffeners' edges as mentioned in the provisions of EN 1993-1-9 [1]. Partial penetration, fulfilling the conditions of the code, of the stiffener to deck plate welding is now easily obtained and generally the penetration is complete. The use of open section stiffeners has become rare and is often considered as uneconomical.

Subsequently, open crossbeam-stiffeners are welded to the longitudinally stiffened deck plate. The intersection of both types of stiffeners rises the matter which of both types should be interrupted. The interruption of the longitudinal stiffeners causes its weakening and common practice points out that the crossbeam webs suffer least from this weakening. Hence the webs are provided with cutouts and generally also with additional cope holes. The stress concentrations and subsequent 
fatigue resistance of these cope holes are the main topic of this paper. In particular, the effect of edge clamping on the stress concentrations is being explored.

\section{FATIGUE RESISTANCE}

\subsection{Critical locations}

The aforementioned rather conventional type of OSD, shows a number of weaknesses, which are already well-known. With reference to Fig. 1 the following locations are prone to reduced fatigue resistance. The butt welds in the deck plate (1) are rarely critical, since longitudinal stress variations are seldom important. The same applies for transverse stresses in the deck plate. These butt welds are mostly one-sided, but because of the low plate thickness full penetration is achieved. The stiffener-to-deck plate weld (2) on the contrary is mostly critical. EN 1993-1-9 [1] is rather strict on this, imposing fatigue class $71 \mathrm{MPa}$. More extensive research [2] based on fracture mechanics, contradicts this.

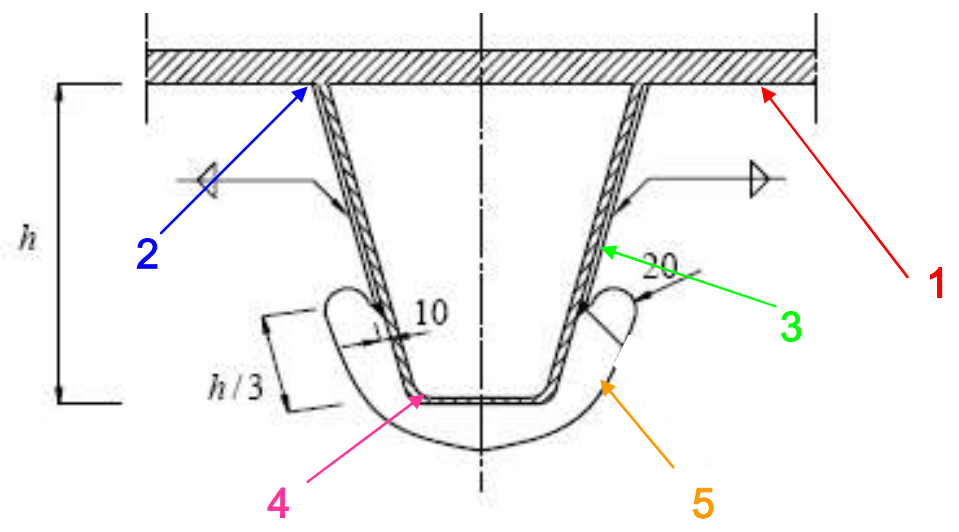

Fig. 1. Fatigue weak spots in OSD

The connection of the stiffener to the crossbeam web (3) transfers the reaction of the load on the stiffener to the crossbeam. In addition, concentrated loads appearing above this area are also transferred through these welds. If the weld length is sufficient $(180-200 \mathrm{~mm})$, the vertical stress flow seldom renders a fatigue problem. However the longitudinal stress in the stiffener web is disturbed. According to EN 1993-1-9 the category is $80 \mathrm{MPa}$.

The butt welds in the stiffener itself are of 2 possible types. Either they are made before fixing the folded plate to the deck plate and can be placed double-sided. However this is useless, since the thickness of $8 \mathrm{~mm}$, welded from one side will certainly result in full penetration. Alternatively, the welds are placed on the construction site on backing strips and belong to category $71 \mathrm{MPa}$. Hence the location of site joints must be chosen accurately in those sections where the stress variations are relatively low.

\subsection{Cutouts and cope-holes}

Finally, the cope holes are flame-cut or with modern equipment such as laser or plasma. Any design should include a model to determine the maximum stress types at the edge of the cope hole, rather than apply framework models as mentioned in table 8.8 of Eurocode EN 1993-1-9.

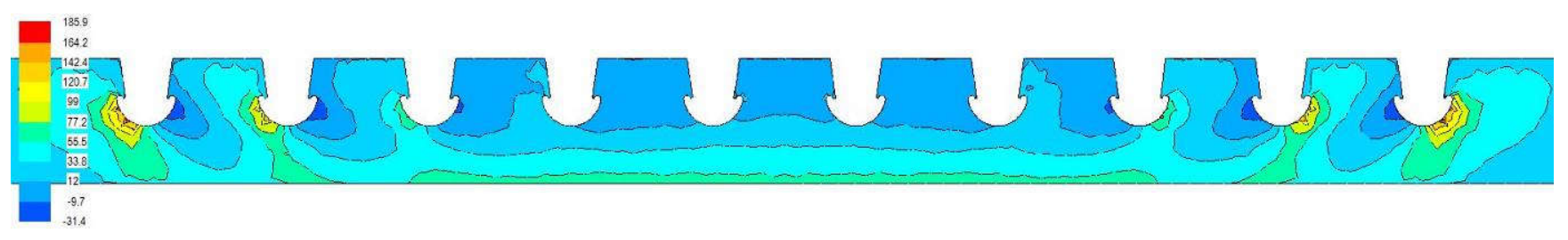

Fig. 2. Stress concentrations at cope-hole edges

It has been sufficiently proven that the framework model is unable to grasp the stress concentrations at this location. If a relevant detailed model is used, the stress concentrations can be determined 
accurately and hot spot stresses are obtained, the fatigue resistance may well be compared to the category for cut plates of $140 \mathrm{MPa}$. Since both normal stresses and shear are appearing at the cope hole edge, the principal stresses should be considered in this comparison.

Fig. 2 shows mainly normal stress and shear stress near the edge appears in a somewhat lower area of the crossbeam web. It may be observed that the critical cope holes are the ones closest to the end members and do not appear below tracks or near the bridge centre. Fig. 3 more specifically shows the distribution of stress concentrations. Obviously, the principal stresses are considered. Towards the crossbeam support a tensile area is found, whereas the opposite edge of the cope hole is subjected to compression. The tensile stress is generally lower than the compression. However both are fostering the initiation of fatigue cracking.

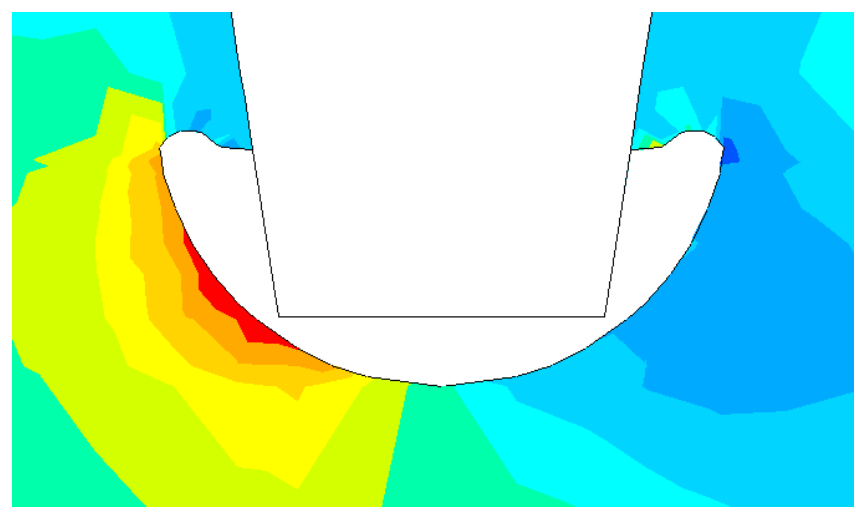

Fig. 3. Detailed stress concentrations

In section 4.1 the 3 causes of these stress concentrations, being the effects of the vertical wheel pressure, shear itself and bending of the crossbeam, are commented more in detail.

\section{VARIABLE HEIGHT OSD}

\subsection{River Dyle Bridge}

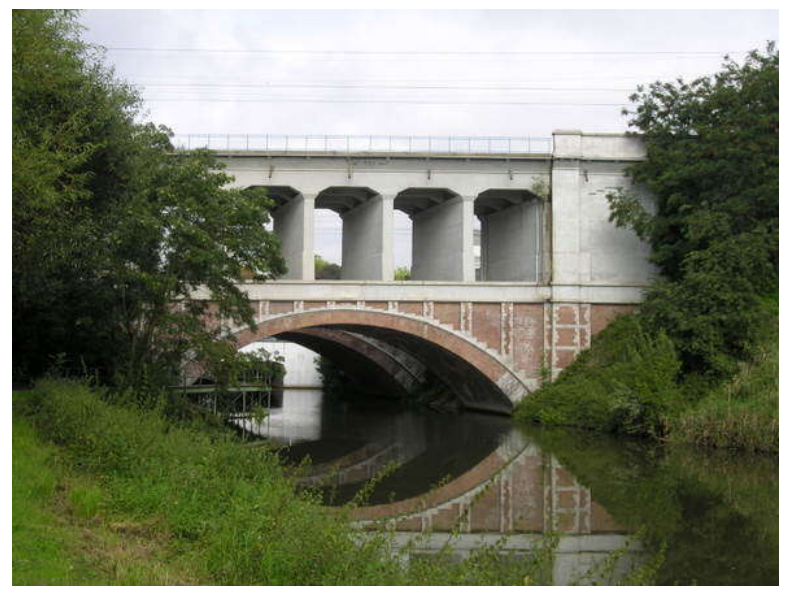

Fig. 4. Brickwork vaulted arch across River Dyle

The project called By-pass of the town of Mechelen included the construction of an additional railway bridge across river Dyle, accommodating new tracks for faster passage through this location. The original site is dominated by a brickwork vaulted arch (Fig. 4), in the 30's it received a concrete superstructure, thus allowing to rise the track level. Combined with the river banks, this structure has been listed as a monument.

The design of the new bridge had to comply with this environment of a slow running river, reflecting the old structure, fishing banks and spontaneous nature. A $50 \mathrm{~m}$ span steel structure of 
lateral curved tubes, connected by OSD was designed. The lateral $1.55 \mathrm{~m}$ outside diameter tubes are slightly bent to introduce thrust force. In addition they are turned inwards to increase the clamping effect of the crossbeams. The arch tubes are clamped in the concrete abutment, using various types of connectors for the combined clamping actions [3].

The structural idea was to refer to the arch shape, and to obtain reflection of the image of the OSD in the water. As the project is still in progress, Fig. 5 does not show yet any reflection and the basic ideas have not become clear. In addition, a third concrete bridge was built in the front. In future, this may obstruct the intended view.

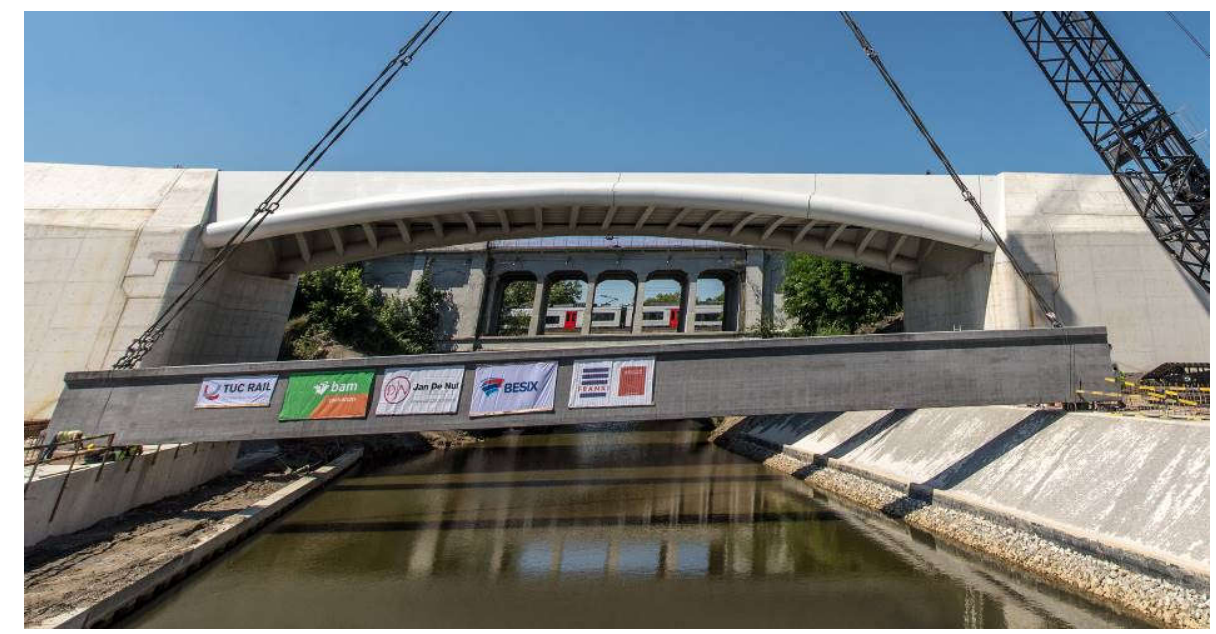

Fig. 5. River Dyle Bridge

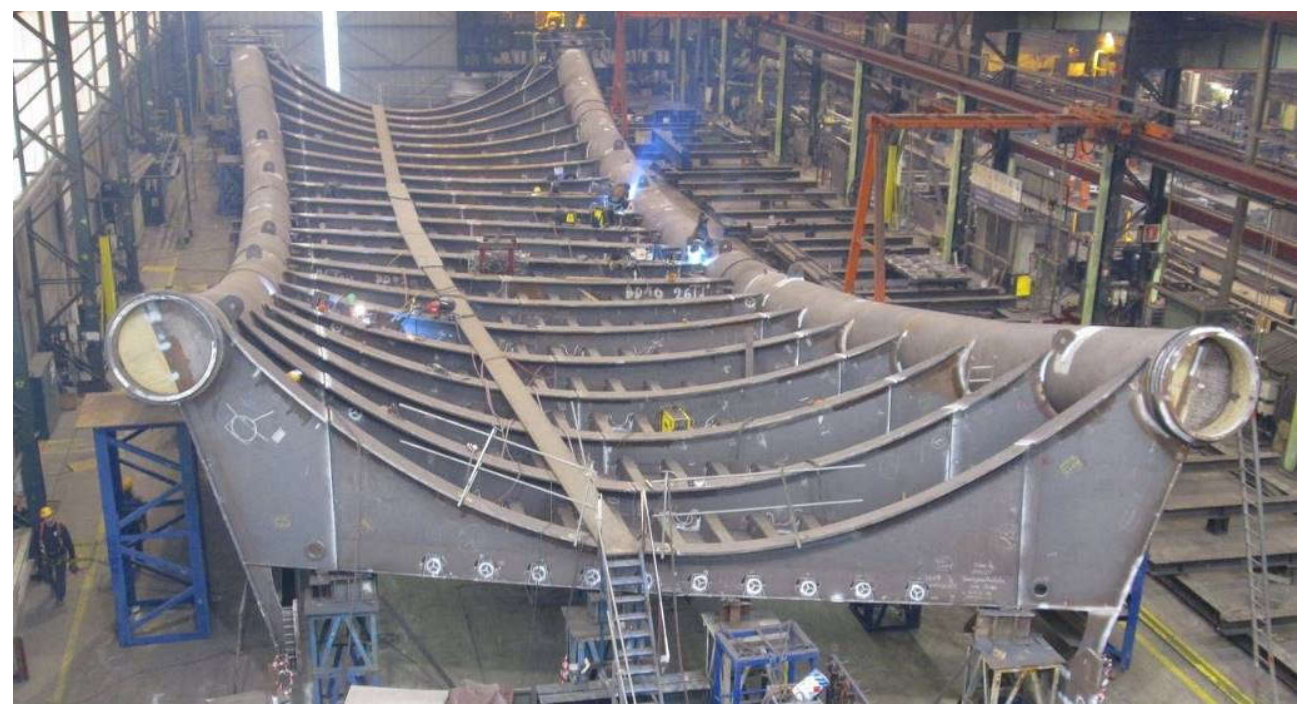

Fig. 6. Workshop assembling of Dyle Bridge

The OSD of the River Dyle Bridge has variable height, both along the bridge axis as in the transverse sense. The latter means the crossbeams also have variable height. This also complies with the inclination of the main tubes and creating a light thrust force. Obviously, the variable height of crossbeams contradicts with a main asset of OSD to minimize the structure's depth.

The lower part of the bridge creates the particularly complicated view as seen in the workshop picture of Fig 6 where it is assembled upside down. The structure was assembled on site in 3 parts for the tube and in 9 parts for the deck.

\subsection{OSD details}

The deck plate has a constant thickness of $15 \mathrm{~mm}$, the closed-section stiffeners having $320 \mathrm{~mm}$ height and $8 \mathrm{~mm}$ thickness. The distance between stiffeners is $0.75 \mathrm{~m}$, the spacing between 
crossbeams being $2 \mathrm{~m}$. The main characteristics of the crossbeam webs are indicated on Fig. 7. At the centre, the height of the web is limited to $587 \mathrm{~mm}$. This total section is reduced by the cope hole to $237 \mathrm{~mm}$. Obviously, this is considerably lower than was used in more than 20 orthotropic plated railway bridges in Belgium [4], the cope hole being located exactly in the area of large shear. At the crossbeam ends, the web height varies from 602 to $2480 \mathrm{~mm}$ since as can also be seen in Fig. 6 . The remaining web height then varies from 252 to $2130 \mathrm{~mm}$. This area is more critical for stress concentrations at the edge of the cope hole. Thus, the central crossbeams are the most prone to stress concentrations and subsequent fatigue.

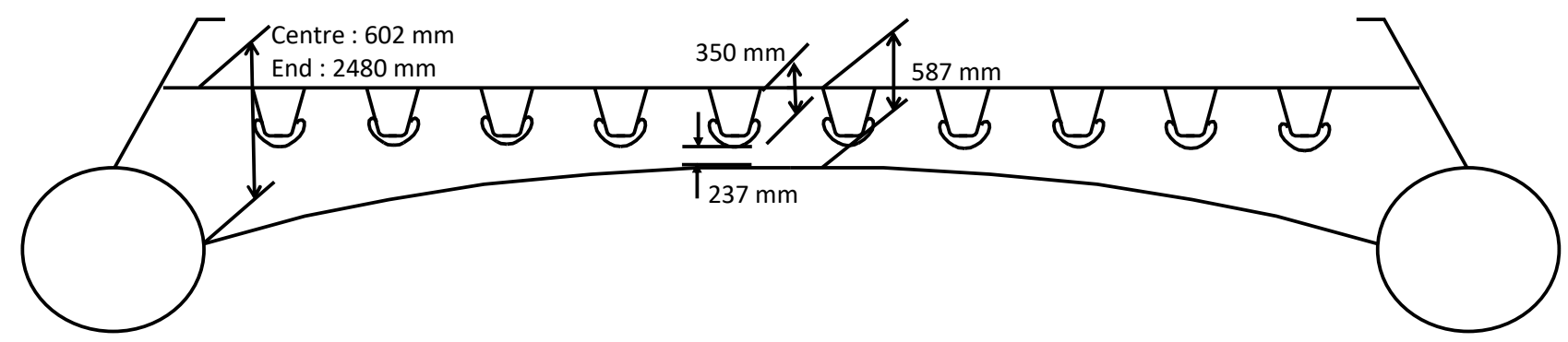

Fig. 7. OSD details and dimensions

\section{FATIGUE CONDITIONS AND CLAMPING}

\subsection{Forces through crossbeam web}

To clarify the mechanism of stress concentrations at the cope hole edge, Fig. 8 may be considered. Vertical wheel loads $\mathrm{W}$ are transmitted to the web and must disperse, albeit the distribution is disturbed by the cope hole. Part of the wheel load is transferred to the closed-section stiffener web. The latter also introduces a vertical reaction, thus increasing the vertical compression in the web. These forces mainly introduce vertical compression stress. In addition, shear force is introduced, which may vary between cross-sections if a wheel force is appearing, as in the case of Fig. 8

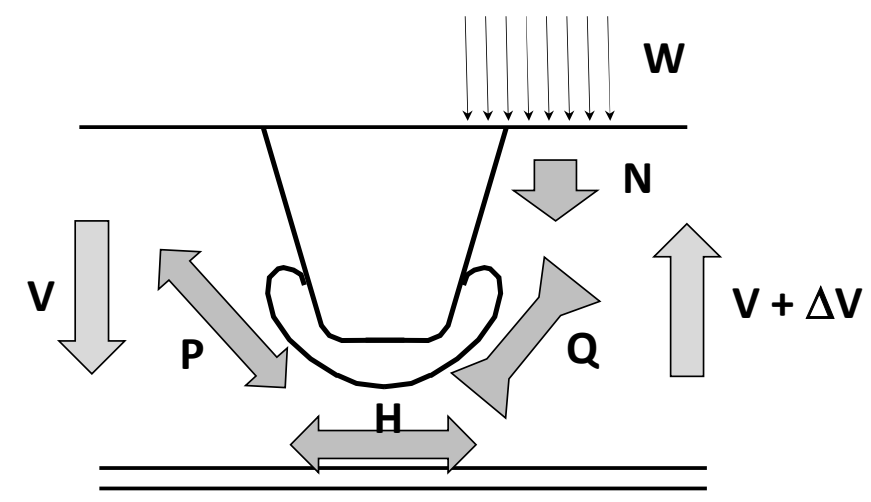

Fig. 8. Various forces influencing stress concentration

If the shear force is undisturbed by vertical loads, it causes equal tensile and compression forces $\mathrm{P}$ and $\mathrm{Q}$, in the vicinity of the free cope hole. In principle, $\mathrm{P}$ and $\mathrm{Q}$ should have equal values and opposite sign. Combined with the vertical effect, the compression force $Q$ should exceed the value of $\mathrm{P}$. In addition, a bending moment exists in the crossbeam. The tensile force $\mathrm{H}$, caused by this bending moment, is passing through the remaining lower part of the crossbeam.

Both the effects of the wheel load and of shear are invariable to possible clamping of the crossbeam ends by the main girder tubes as in Fig. 7. The only part that would be sensitive to clamping moments of the crossbeam ends is the tensile force H. A larger clamping ratio will reduce this tensile force and probably result in smaller compression of Q and possibly increase of the value of $\mathrm{P}$. Since, even if no wheel load $\mathrm{W}$ is acting in a particular area, the concentration of compression 
stress tends to be higher than of tensile stress, partial or complete moment clamping of the crossbeam ends may result in lower stress concentration and thus higher fatigue resistance.

\subsection{Parametric simulations}

The clamping effect can also be expressed as the distance between those points where the bending moment in the crossbeam equals 0 . This allows simulating various degrees of clamping and renders results independent of the main girder torsion stiffness or other relevant parameters. Fig. 8 shows the peak value stresses in the web of the central crossbeam of River Dyle bridge. The drawn line represents the compression stresses (the negative sign being removed) corresponding to Q, whereas the dotted line gives tensile stresses, corresponding to $\mathrm{P}$.

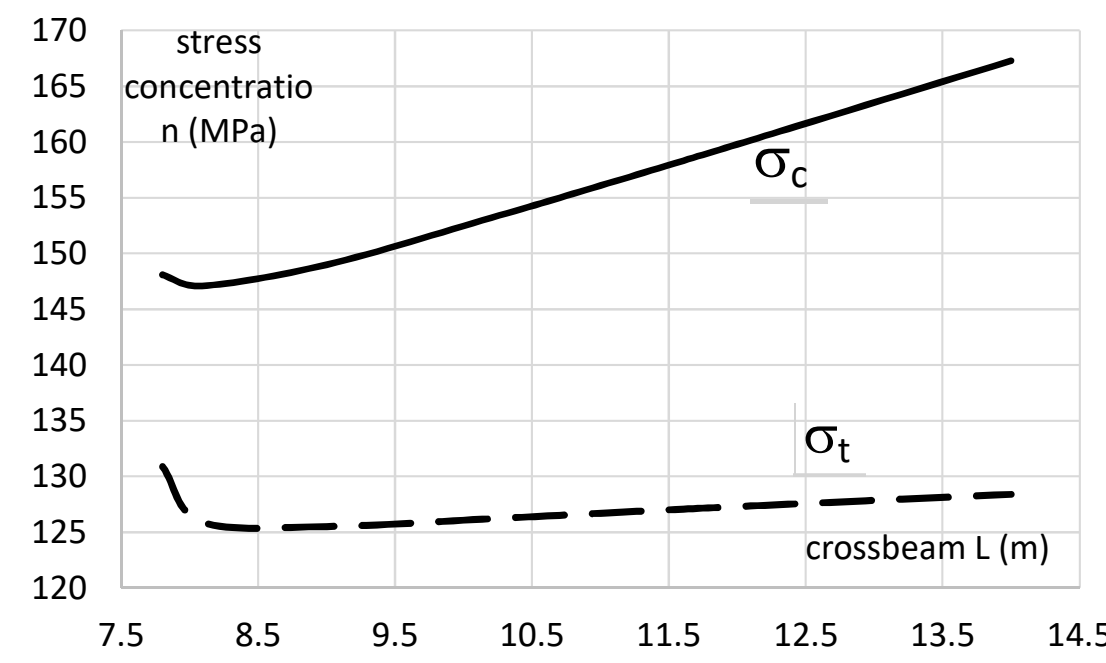

Fig. 8. Compression and tensile stress concentrations versus $\mathrm{L}$

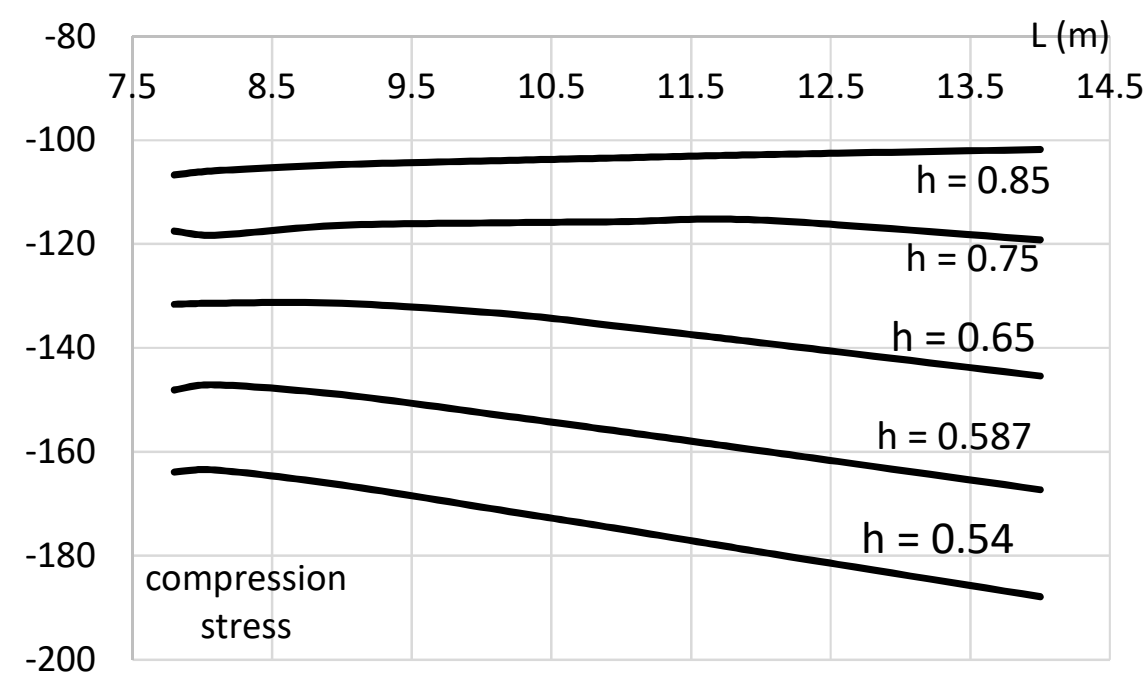

Fig. 9. Compression stress concentrations versus crossbeam height

The extreme value to the left is rather inadequate, since the crossbeam is so short that interference exists between the effect of the cope holes and the support of the crossbeam. Hence, the conclusion is that shorter crossbeams, or a high clamping effect results in lower compression stress as well as tensile stress. The reduction of the latter however is rather moderate. Comparing the data for $\mathrm{L}=14$ or $\mathrm{L}=8$, the decrease of tensile stress equals $2 \%$, whereas it reaches $12 \%$ for the more critical compression. In terms of fatigue life time the equivalent values are respectively 10 and $90 \%$. These results demonstrate that the effect of edge clamping may certainly have paramount influence.

Obviously, the former result may be due to the small height of the crossbeam web. In 3.2 it was mentioned already that the cope hole is located exactly in the area of high shear. The reduction of 
the critical compression stress concentration may be due exclusively to this and the design of the OSD may be poor.

To verify whether the reduction is found for other values of the crossbeam height, the latter was varied, together with L. In the diagram of Fig 9 the compression stress concentrations are given. This diagram clearly indicates that for large crossbeam height, the effect may be reversed. This occurs for rather large values of $\mathrm{L}$, which are out of the practical range.

The latter is due to the fact that the cope hole's location has shifted to the bending compression area. The mechanism as explained in 3.1 therefore is confirmed. This can also be seen for the tension stress, since a minimum was reached for $\mathrm{L}=10.2 \mathrm{~m}$ and the values are increasing after reaching the minimum value.

It may be concluded that the decreasing of critical stress concentrations by partial clamping of crossbeam ends is more effective for low crossbeam height and certainly stands if the cope hole is located in the area of tension due to bending.

\subsection{Influence length}

In order to determine one of the relevant dynamic factors for railway loads, both the conventional $\Phi$ and the real train effect factor $\varphi$ the length of the influence line for stresses in the crossbeams, must be determined. According to Table 6.2 of EN 1991-2 [5] the characteristic length L $\Phi$ equals twice the crossbeam span. However, this value is sometimes criticised and replaced by twice the crossbeam spacing $+3 \mathrm{~m}$. The influence length was determined from the complete model of the river Dyle Bridge. This is shown in Fig 10.

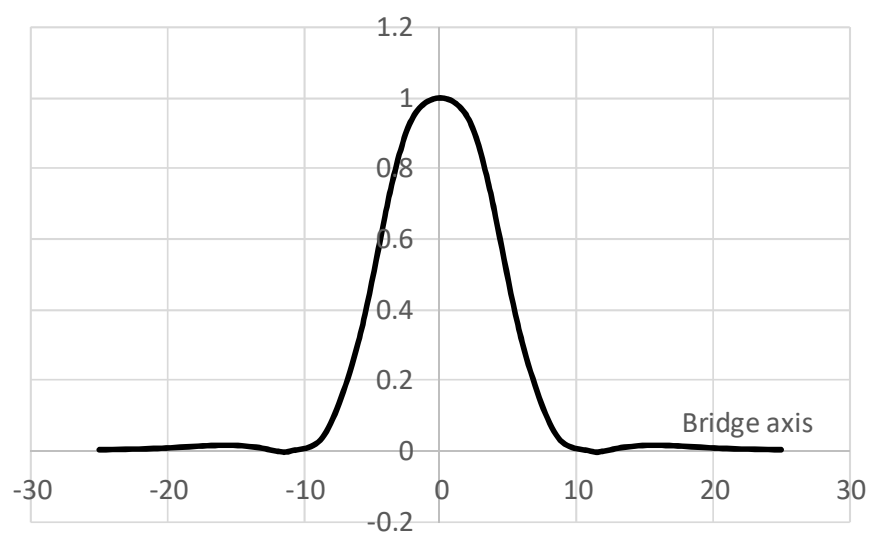

Fig. 10. Influence length for stress concentrations cope hole

The influence length corresponds to $21 \mathrm{~m}$, whereas the crossbeam length is $10.2 \mathrm{~m}$. Hence, the value of table 6.2 corresponds rather well with this result. In this particular case it meant that the conventional dynamic factor $\Phi$ is lowered from 1.410 to 1.154 .

\section{SUMMARY AND ACKNOWLEDGMENT}

It was implied that complete or partial clamping of the ends of crossbeams might influence the magnitude of the stress concentrations at the edges of the cope holes in the webs of an OSD. This may occur in the particular case of the river Dyle railway Bridge in Mechelen. More careful analysis confirms this, since in particular the compression stress concentration at the cope hole edge is decreased. The reduction of these compression stress concentrations may substantially increase the fatigue resistance. However, the effect is mainly due to the tensile bending stress of the crossbeam and thus varies with the span.

Variation of the crossbeam height confirmed that the reduction of peak value stresses exists if the cope hole is located in an area of bending tension stress. Increasing of the crossbeam height may erase the effect completely. Therefore the reduction of stress concentrations due to clamping applies only to low height crossbeams. 


\section{REFERENCES}

[1] EN 1993-1-9 : Eurocode 3 : Design of steel structures - Part 1-9 : Fatigue. CEN May 2005 (+AC 2006)

[2] Nagy, W., Schotte, K., Van Bogaert, Ph., De Backer, H., "Fatigue strength application of fracture mechanics to orthotropic steel decks", Advances in Structural Engineering, Vol 19 Nr 11 Nov 2016 pp 1696-1709.

[3] Van Bogaert, Ph., "Torsion Clamping by Prestressing Bars of an Arch Bridge in Concrete Abutment." In Fib Symposium, Engineering a Concrete Future: Technology, Modeling \& Construction, Proceedings, FIB 2013 pp 447-450

[4] Van Bogaert, Philippe. (2003). Steel tied arch railway bridge. Structural engineering international, 13(1), 22-24

[5] EN 1991-2 : Eurocode 1 : Actions on structures - Part 2 : Traffic loads on bridges. CEN Sept 2003. 